\title{
Fractal fluctuations in quantum integrable scattering
}

\author{
Italo Guarneri $^{(a, b, c)}$, and Marcello Terraneo ${ }^{(a, b)}$ \\ ${ }^{(a)}$ International Center for the Study of Dynamical Systems, \\ Università degli Studi dell'Insubria, Via Valleggio 11, 22100 Como, Italy \\ ${ }^{(b)}$ Istituto Nazionale per la Fisica della Materia, Unità di Como, Via Valleggio 11, 22100 Como, Italy \\ ${ }^{(c)}$ Istituto Nazionale di Fisica Nucleare, Sezione di Pavia, Via Bassi 6, 27100 Pavia, Italy
}

(November 4, 2018)

\begin{abstract}
We theoretically and numerically demonstrate that completely integrable scattering processes may exhibit fractal transmission fluctuations, due to typical spectral properties of integrable systems. Similar properties also occur with scattering processes in the presence of strong dynamical localization, thus explaining recent numerical observations of fractality in the latter class of systems.
\end{abstract}

PACS numbers: 05.45.Df, 05.40.-a, 05.45.Mt

Scattering processes dominated by a statistically large number of metastable states display reaction rates which depend on energy or other control parameters in complicated ways. Reaction curves (i.e., curves obtained by plotting cross sections versus the relevant parameters) demand statistical description. This approach, originated in nuclear physics, has given rise to the theory of quantum chaotic scattering [1]. Classical chaotic dynamics plays an important role therein [2], with applications in many fields, including the study of mesoscopic conductance fluctuations [3].

In the presence of complicated reaction curves, a natural question is about their fractality. The latter is not meant in the strict mathematical sense, but rather as a property to be possibly observed on a wide range of resolution scales. Reaction curves probe the location of resonance poles in the complex energy plane, so their fluctuation properties - including fractality - are encoded in the distribution of such poles. In particular, the lack of smoothness required by fractality can only be produced by resonance poles clustering in the vicinity of the real energy axis. This excludes fractal reaction curves in quasi-classical cases when the underlying classical dynamics is completely chaotic (i.e, uniformly hyperbolic), because the quantum resonances are then concentrated away from the real axis. This reflects the exponential decay in time exhibited by such systems over long time scales. A dynamical signature of poles clustering near the real axis is instead slow algebraic decay of the survival probability inside the interaction region. On the classical level, such slow decay is in particular exhibited by systems with a mixed phase space, endowed with a hierarchical structure of stable islands [4]. On such grounds, fractal fluctuations were predicted by Ketzmerick for the quasi-classical transmission of electrons through mesoscopic cavities [5]. This prediction has received numerical [6] and experimental [7] support. However, fractal fluctuations have been numerically observed also in $2 \mathrm{~d}$ tight-binding models of quantum dots, where the relation to classical dynamics is unclear [8], and even in models where this relation is irrelevant [9], due to strong quantum localization on one hand, and to absence of significant classical critical structures on the other. It therefore appears that fractality of reaction curves is not strictly associated with critical structures in the classical phase space.

The issue of fractality in the remaining major class of dynamical systems, namely the integrable ones, has not yet been investigated [10]. This is the main purpose of this Letter. We first identify special statistical properties of resonance poles, which afford fractal reaction curves. This we do in absolute generality, without assuming integrability or other special properties of the quantum system. We then argue that such properties are optimally exhibited by suitable, completely integrable processes, thanks to a well known generic property of integrable systems, that their energy levels share some of the properties of a random sequence [11. We use a textbook scattering problem to confirm our theory with numerical data. Finally we note that the same properties are also typical of fully chaotic, yet strongly localized systems, and thus we explain the results of ref. [9].

We consider a weakly open quantum system, with scattering resonances at $E_{j}-i \Gamma_{j} / 2$ and the real energies $E_{j}$ arranged in increasing order. The energy dependence of a typical cross section $T(E)$ consists of a smooth background plus a resonant part, which we write in the form:

$$
T_{r}(E)=\sum_{j} c_{j} \frac{\Gamma_{j}^{2}}{\left(E_{j}-E\right)^{2}+\Gamma_{j}^{2}}
$$

with $c_{j}$ slowly varying with $j$. We restrict within an energy interval $\left(E_{0}-W, E_{0}+W\right)$, and we assume $\epsilon \ll W \ll$ $E_{0}$, where $\epsilon$ is the mean level spacing. To perform fractal analysis of the graph of $T_{r}(E)$ vs $E$ we divide the interval $\left(E_{0}-W, E_{0}+W\right)$ into subintervals $\Delta_{k},(k=1,2, \ldots, M)$ of equal size $\delta \propto 1 / M$. Upon each subinterval we pile up squares of side $\delta$, and denote by $N(\delta)$ the total number of squares met by the graph of $T_{r}(E)$. Algebraic scaling $N(\delta) \propto \delta^{-f}$ with $f>1$ between scales $\delta_{\min } \ll \delta_{\max }$ signals that in between such scales the graph exhibits a 
fractal (box-counting) dimension $f$. In order to determine $f$ we compute

$$
\frac{\log (N(\delta))}{\log \left(\delta^{-1}\right)} \approx 2+\frac{\log \left(M^{-1} \sum_{k} \sigma_{k}\left(T_{r}\right)\right)}{\log (M)} \approx 2-\frac{\log \left\langle\sigma\left(T_{r}\right)\right\rangle}{\log (\delta)}
$$

where $\sigma_{k}\left(T_{r}\right) \geq 0$ is the maximal excursion of $T_{r}(E)$ within the $k$-th interval. We next assume the following. First, in the given energy interval, the frequency $P(\Gamma)$ of widths less than $\Gamma$ scales like $P(\Gamma) \sim a \Gamma^{1-\alpha}$ at $\epsilon<\Gamma<\bar{\Gamma}$, with $\bar{\Gamma}$ the mean resonance width, and $0<\alpha<1$. Second, both $\Gamma_{j}$ and $E_{j}$ form uncorrelated sequences. Finally, resonances are strongly overlapped, in the sense that $\epsilon \ll \bar{\Gamma} \ll W$. Then, at $\epsilon \ll \delta \ll \bar{\Gamma}$, the fluctuation of $T_{r}$ in an interval $\Delta_{k}$ is mostly due to many tiny resonant peaks which are centered inside the interval and are narrower than $\delta$. Every such peak contributes a Lorentzian term in (ID), and the mean square oscillation of this term as $E$ ranges in $\Delta_{k}$ is $\sim \delta^{-1} P(\delta)^{-1} \int_{0}^{\delta} d P(\Gamma) \Gamma \sim 1$. There are $n(\delta) \sim \epsilon^{-1} \delta P(\delta)$ such peaks; as they contribute uncorrelated oscillations, we estimate $\left\langle\sigma\left(T_{r}\right)\right\rangle \sim \sqrt{n(\delta)} \sim \epsilon^{-1 / 2} \delta^{1-\alpha / 2}$. Therefore, $f=1+\alpha / 2$ in a range $\delta_{\min }<\delta<\delta_{\max }$, with $\delta_{\max } \sim \bar{\Gamma}$, and $\delta_{\min }$ roughly estimated by $n\left(\delta_{\min }\right) \sim 1$, that is, $\delta_{\min } \sim(\epsilon / a)^{1 /(2-\alpha)}$.

Fluctuating cross sections may also be generated at fixed energy, by varying other parameters, as in the case of magnetoresistance fluctuations in mesoscopic physics. The above analysis carries over to such fluctuations on replacing energy by the relevant parameter, provided that the above assumptions remain satisfied.

The above described conditions are met in some physically relevant situations. Uncorrelated sequences of energy levels are a distinctive feature of generic integrable systems [11]. We then surmise fractal reaction curves for completely integrable scattering processes, provided they display a slow decay, leading to an inverse power law distribution of $\Gamma$ 's. We shall presently describe an explicit example of such a process.

We consider the quantum dynamics of a particle of unit mass moving inside the infinite strip $0 \leq x \leq L_{x}$ in the $(x, y)$ plane, with tunneling barriers at $y= \pm L_{y} / 2$. The Hamiltonian is

$$
H=-\frac{\hbar^{2}}{2} \Delta+\hbar \sigma \delta\left(y-L_{y} / 2\right)+\hbar \sigma \delta\left(y+L_{y} / 2\right)
$$

We use periodic boundary conditions at $x=0, x=L_{x}$. The Dirac delta functions enforce additional boundary conditions: $\partial_{y} \psi(x, y+)-\partial_{y} \psi(x, y-)=2 \sigma \hbar^{-1} \psi(x, y)$ at $y= \pm L_{y} / 2$. The physical model is a rectangular billiard, whence the particle can escape into semi-infinite leads, by tunneling through the two horizontal sides. For $|y|>L_{y} / 2$ eigenfunctions are $u_{E, m}^{ \pm}(x, y)=\phi_{m}(x) \theta_{k}^{ \pm}(y)$, $\phi_{m}(x)=\exp \left(2 \pi i m x / L_{x}\right), \theta_{k}^{ \pm}(y)=A^{ \pm}(k) \exp (i k|y|)+$ $B^{ \pm}(k) \exp (-i k|y|)$ and $2 E / \hbar^{2}=k^{2}+4 \pi^{2} m^{2} / L_{x}^{2}$, with \pm denoting the upper $\left(y>L_{y}\right)$ and the lower $\left(y<-L_{y}\right)$ lead respectively. For given $E>0$ there are a finite number of open scattering channels labelled by the integer $m,|m| \leq \operatorname{Int}\left(L_{x}(\pi \hbar)^{-1} \sqrt{E / 2}\right)$, and by the lead label \pm . The coefficients $A^{ \pm}(k)$ are related to $B^{ \pm}(k)$ by the scattering matrix. Scattering resonances are located at complex values of energy $z_{n, m}=E_{n, m}-i \Gamma_{n} / 2=$ $2 \pi^{2} m^{2} \hbar^{2} / L_{x}^{2}+k_{n}^{2} \hbar^{2} / 2,(n=1,2, \ldots)$, where $k_{n}$ are the complex roots of the equations:

$$
e^{i k L_{y}} \pm 1 \mp i k \hbar \sigma^{-1}=0
$$

The following asymptotic formulae can be computed by using Lagrange's theorem on the inversion of analytic functions:

$$
\begin{gathered}
E_{n, m}=E_{n, m}^{0}-\frac{\hbar v_{n}^{2}}{L_{y} \sigma}\left\{\frac{\sigma}{v_{n}} \arctan \left(\frac{v_{n}}{\sigma}\right)+O\left(\frac{\hbar}{\sigma L_{y}}\right)\right\} \\
\Gamma_{n}=\frac{\hbar v_{n}^{3}}{L_{y} \sigma^{2}}\left\{\frac{\sigma^{2}}{v_{n}^{2}} \log \left(1+\frac{v_{n}^{2}}{\sigma^{2}}\right)+O\left(\frac{\hbar}{\sigma L_{y}}\right)\right\}
\end{gathered}
$$

where $v_{n}=n \pi \hbar / L_{y}$ is the velocity in the $n$-th vertical mode of the closed rectangle, and $E_{n, m}^{(0)}=2 \hbar^{2} \pi^{2} m^{2} / L_{x}^{2}+$ $v_{n}^{2} / 2$ are the eigenvalues of the closed $(\sigma=\infty)$ billiard. The 1st term on the rhs of the 2nd eq.(3) is the decay rate of a classical billiard ball inside the closed rectangle, with velocity $v_{n}$ in the $y$-direction, and absorption probability at $y= \pm L_{y} / 2$ equal to the transmission coefficient for a plane wave through on a $\delta$ barrier, given by $v_{n}^{2}\left(\sigma^{2}+v_{n}^{2}\right)^{-1}$. We assume $\hbar \ll \sigma L_{y}$ and thereby neglect $O\left(\hbar / \sigma L_{y}\right)$ corrections in (3). The statistics of real parts of resonances is then like the energy level statistics of an integrable system, with a mean level spacing only different by corrections of order $\left(\hbar / \sigma L_{y}\right)^{3}$ from that of the closed billiard: $\epsilon=2 \pi \hbar^{2}\left(L_{x} L_{y}\right)^{-1}$. The correction to the closed billiard levels in (3) lifts possible degeneracies due to commensurate geometry.

In an energy interval $\left(E_{0}-W, E_{0}+W\right), \epsilon \ll W \ll E_{0}$, the smooth (Thomas-Fermi) part of the integrated $P(\Gamma)$ distribution is computed from a microcanonical distribution of classical billiard trajectories at energy $E_{0}$, each with a decay rate $\Gamma$ (3). This quasi- classical distribution has a mean $\bar{\Gamma}_{E_{0}} \simeq \hbar \sigma^{-2} L_{y}^{-1} E_{0}^{3 / 2}$, and behaves like $a \Gamma^{1 / 3}$ at small $\Gamma$, with $a=\left(\pi^{2} E_{0} / 2\right)^{-1 / 2}\left(L_{y} \sigma^{2} / \hbar\right)^{1 / 3}$. The same behaviour can be assumed for the quantal distribution, provided that $E_{0} \leq \sigma^{2}$, and that $\epsilon \ll \bar{\Gamma}_{E_{0}}$. The former condition ensures $\Gamma^{1 / 3}$ behaviour of the quasiclassical distribution in a range of $\Gamma$-values comparable to $\bar{\Gamma}_{E}$. Together with the latter condition it ensures that the quasi-classical behaviour is observed over a statistically significant number of resonances. Reflecting generic properties of energy spectra of integrable systems, the real parts of resonances, arranged in increasing order, form an essentially uncorrelated sequence. At fixed quantum number $m$, they form an ordered ladder, but the superposition of a large number of different, uncorrelated ladders results in a Poisson-like statistics. On the same grounds we assume uncorrelated resonance widths $\Gamma$, too. 
The transmission amplitude at energy $E$ from the lower $m$ - channel to the upper $l-$ channel is:

$$
S_{m-, l+}(E)=\frac{k^{2}(E, m) \hbar^{2}}{\sigma^{2} e^{2 i k(E, m) L_{y}}+(k(E, m) \hbar+i \sigma)^{2}} \delta_{m l}
$$

where $k(E, m)=\sqrt{2 E \hbar^{-2}-4 \pi^{2} m^{2} L_{x}^{-2}}$. A computation shows that the residue of (A) at a resonance pole is $\sim-i \Gamma / 2$ at small $\Gamma$. Hence, the resonant part of the total transmission coefficient:

$$
T(E)=\sum_{m}\left|S_{m-, m+}(E)\right|^{2}
$$

(the sum being over all open channels at energy $E$ ) has the form (1) apart from a slowly varying factor. The smooth part is $\propto E^{5 / 2} / \sigma^{4}$. Collecting various estimates we see that at $\hbar /\left(\sigma L_{y}\right) \ll 1, \hbar^{2} / L_{y}^{2} \ll W \ll E_{0}<\sigma^{2}$, $E_{0}^{3 / 2} L_{x} \hbar^{-1} \sigma^{-2} \gg 1$ the assumptions of our general argument are satisfied, hence the graph of $T(E)$ vs $E$ in $\left(E_{0}-W<E<E_{0}+W\right)$ should be fractal with dimension $f=4 / 3$, over scales intermediate between $\delta_{\min } \sim 1.8 \epsilon^{3 / 4} \bar{\Gamma}_{E_{0}}^{1 / 4}$ and $\delta_{\max } \sim \bar{\Gamma}_{E_{0}}$.

A numerically computed graph of $T(E)$ is shown in Fig.1 (lower). The corresponding fractal analysis is shown in Fig.2 and fully confirms the theory. In that case, the above estimates for $\delta_{\min }, \delta_{\max }$ give $10^{1.9}$ and $10^{3.9}$ respectively.

A generalization of the above model allows for the investigation of parametric fluctuations. It is obtained by using boundary conditions $\psi(0, y)=e^{i \phi} \psi\left(L_{x}, y\right)$. This is equivalent to a particle moving on a cylinder, with axis in the $y$ - direction, enclosing a magnetic flux $\phi$. This problem is still completely integrable. Replacing $m$ by $m-\phi /(2 \pi)$ throughout the equations derived at $\phi=0$ yields the corresponding theory. At fixed energy $E$, the total transmission fluctuates as $\phi$ is varied, in the manner illustrated in Fig.1 (upper). The theory of such fluctuations is completely parallel to the one we have described for fluctuations vs energy at fixed $\phi=0$. Resonances depend on $\phi, z_{n, m}=z_{n, m}(\phi)$, and the complex values of $\phi$ solving the equations $z_{n, m}(\phi)=E$ define resonance poles in the complex $\phi$-plane. Omitting computational details, the real parts of such poles are distributed in $[0,2 \pi]$ with a mean spacing $\epsilon_{\phi} \sim \pi^{2} \hbar \sqrt{2 / E} / L_{x}$. The distribution of their widths $\Gamma_{\phi}$ behaves like $\Gamma_{\phi}^{1 / 3}$ at small $\Gamma_{\phi}$, with a mean $\sim L_{x}^{2} \bar{\Gamma}_{E} \epsilon_{\phi} /\left(8 \pi^{2} \hbar^{2}\right)$. Our general discussion is thus valid for parametric fluctuations, too. In fact numerical data shown in Fig.3 demonstrate the predicted fractal dimension $4 / 3$.
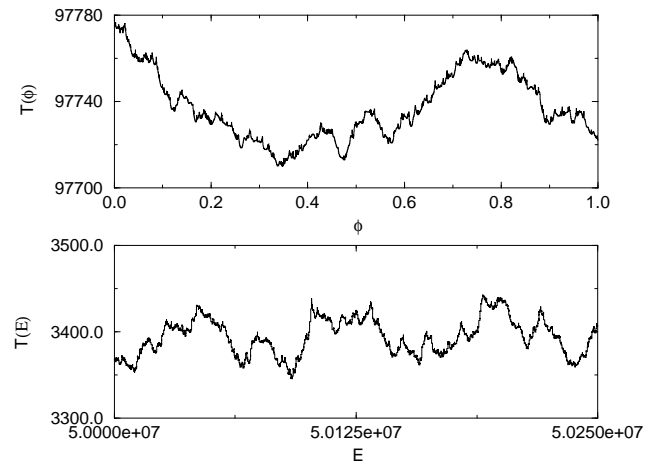

FIG. 1. Total transmission versus flux $\phi$ at fixed energy $E=5 \times 10^{10}$ (upper plot), and versus energy $E$ at fixed flux $\phi=0$ (lower plot), for $\sigma=3.5 \times 10^{5}$ and $\sigma=10^{4}$ respectively. In both cases $\hbar=1, L_{x}=2, L_{y}=0.4$. The energy range in the lower plot is $\left(E_{0}, E_{0}+W\right)$ with $E_{0}=5 \times 10^{7}$, $W=2.5 \times 10^{5}$. Units are arbitrary.

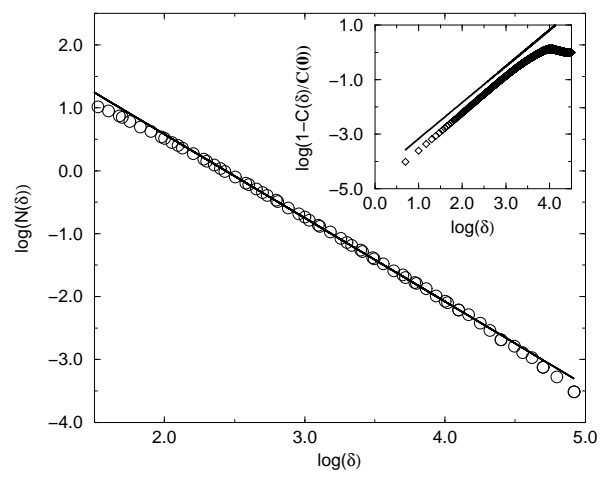

FIG. 2. Fractal analysis of the graph of $T(E)$ vs $E$ shown in the lower fig 1. The meaning of $\delta, N(\delta)$ is explained in the text. The straight line corresponds to fractal dimension $f=4 / 3$. The inset shows the autocorrelation $1-C(\delta) / C(0)$ vs $\delta$. The straight line has slope $4 / 3$.

Both for energy-dependent and for parametric fluctuations we have computed autocorrelations of the fluctuation graphs. For the case of fluctuations vs energy, such correlations are defined by

$$
C(\delta)=\left\langle T^{(f l)}(E) T^{(f l)}(E+\delta)\right\rangle_{E}
$$

where $T^{(f l)}$ is obtained from $T$ by subtracting a smooth, slowly varying part, and the average is taken over the scanned interval of energies. Treating $T^{(f l)}(E)$ as a stationary stochastic process, one easily finds that such correlations behave at small $\delta$ like $C(0)-$ const. $\times \delta^{2 \gamma}$, where $\gamma$ is the scaling exponent of the rms increment of $T(E)$ over intervals of length $\delta:\left\langle|T(E+\delta)-T(E)|^{2}\right\rangle_{E} \propto \delta^{2 \gamma}$ (note that subtracting the smooth part does not alter fractional scaling). Generally speaking, the rms increment is a quite different quantity from the average excursion which enters the definition of the fractal dimension. 
With the present strong statistical properties the two quantities scale in the same way at small $\delta: 2 \gamma=2-\alpha$, so $C(\delta) \sim C(0)-$ const. $\times \delta^{4 / 3}$, as confirmed by numerical data. However fractional scaling of correlations is not in general a sufficient condition for fractality. For instance, correlations in Fig.2 and in Fig.3 exhibit a 4/3 scaling down to small $\delta$ scales below the fractal range. This is because they are still determined by the statistics of narrow, non-overlapped individual peaks, which do not produce fractality any more. On the other hand, on increasing $\delta$ correlation functions depart from the predicted fractional behaviour already at values well within the fractal range, because a larger statistics is needed for increments recorded over a finite $\delta$-grid to sample the distribution of excursions over the same grid.

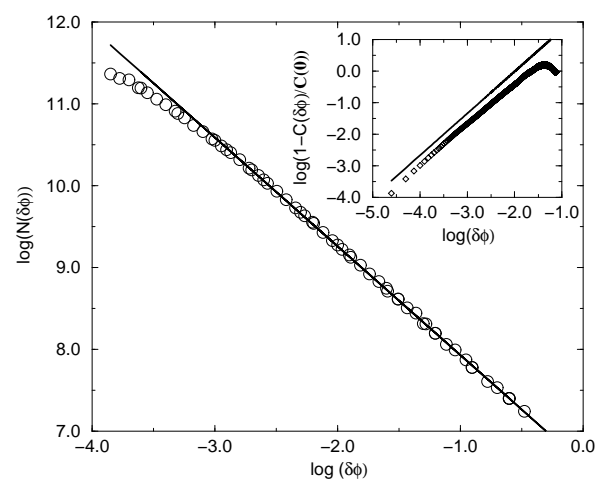

FIG. 3. Fractal analysis of the flux-dependent fluctuations shown in the upper fig.1. The straight line corresponds to $f=4 / 3$. The inset shows the correlation scaling exponent $2 \gamma=4 / 3$.

The work reported in this Letter hinges on the fully general fact, that fractality of scattering fluctuations is, first and foremost, a matter of complex level statistics. As in the case of real level statistics (closed systems), integrable systems have special properties in this respect, and we have in fact demonstrated fractal reaction curves for a completely integrable process. A few remarks are in order about the relevance of this finding:

(1) In completely integrable scattering processes there is no mixing of flux between different channels. Like pseudo-randomness of energy levels, fractality comes of superimposing non-fractal fluctuation patterns from different, uncorrelated channels. Therefore the nature of the reaction curves depends on the relative weight assigned to different channels.

(2) The present result may also be relevant to quasiintegrable systems, which possess large stable components in their phase space. Such components may contribute a significant set of resonances, produced by tunneling through invariant manifolds, with the statistical properties considered in this Letter. Their interplay with critical structures at the border of the stable regions de- mands careful analysis.

(3) uncorrelated energy spectra also occur with fully chaotic systems in the regime of strong quantum localization. A prototype system in this class is the kicked rotor, the quasi-energy spectrum of which has a Poisson-like statistics in case of strong localization 12. For this class of systems, the $\Gamma$ (differential) distribution behaves like $1 / \Gamma$ down to very small scales $[13,14]$. This explains recent findings [9] of parametric fractal fluctuations with dimension $3 / 2$, detected in the survival probability at fixed time, on varying a magnetic flux $\phi$.

Support from MURST Research Project "Chaos and localization in classical and quantum mechanics" is gratefully acknowledged.

[1] Y.V.Fyodorov and H.J. Sommers, J.Math.Phys. 38 (1997) 1918.

[2] U.Smilansky, in the Proceedings of the 1989 Les Houches Summer School on Chaos and Quantum Physics, M.Giannoni, A. Voros and J.Zinn-Justin eds., North Holland, Amsterdam 1991.

[3] R.A.Jalabert, in the Proceedings of the International School of Physics "Enrico Fermi", course CXLIII, G.Casati, I.Guarneri and U.Smilansky eds,. IOS Press (2000), and references therein.

[4] T.Geisel, A.Zacherls and G.Radons, Phys. Rev. Lett.59 (1987), 2503

[5] R.Ketzmerick, Phys. Rev. B54 (1996) 10841; L.Hufnagel, R.Ketzmerick and M.Weiss, Europhys. Lett., 54 (2001) 703.

[6] G.Casati, I.Guarneri and G.Maspero, Phys.Rev.Lett. 84 (2000) 63.

[7] H.Hegger, B.Huckestein, K.Hecker, M.Janssen, A. Freimuth, G.Reckziegel and R.Tuzinski, Phys.Rev.Lett. 77 (1996) 3885; A.P.Micolich, R.P.Taylor, R.Newbury, J.P.Bird, R.Wirtz, C.P.Dettmann, Y.Aoyagi and T.Sugano, J.Phys: Condens. Matter 10 (1998) 1339; A.S. Sachrajda, R. Ketzmerick, C. Gould, Y. Feng, P.J. Kelly, A. Delage, and Z. Wasilewski, Phys.Rev.Lett, 80 (1998) 1948.

[8] E.Louis and J.A.Verges, Phys.Rev. B61(2000) 13014.

[9] G.Benenti, G.Casati, I.Guarneri and M.Terraneo, Phys. Rev. Lett., 87 (2001) 014101

[10] Transport fluctuations in integrable cavities have been studied in detail, but their fractality doesn't appear to have been investigated as yet. See, e.g., ref. [3].

[11] M.V.Berry and M.Tabor, Proc. R. Soc. London, Ser. A, 349, (1976) 101.

[12] M.Feingold, S.Fishman, D.R.Grempel and R.Prange, Phys. Rev. B 31 (1985) 6852.

[13] G.Casati, G.Maspero and D.L.Shepelyansky, Phys. Rev. Lett. 82, (1999), 524.

[14] M.Titov and Y.Fyodorov, Phys.Rev. B61 (2000) 2444; M.Terraneo and I.Guarneri, Eur.Phys.J. B18 (2000),303. 